\title{
3D-Printed Ultra-Broadband Highly Efficient Out-of-Plane Coupler for Photonic Integrated Circuits
}

\author{
Matthias Blaicher ${ }^{1,2}$, Muhammad Rodlin Billah ${ }^{1,2}$ Tobias Hoose $^{1,2}$, Philipp-Immanuel Dietrich ${ }^{1,2}$, \\ Andreas Hofmann ${ }^{3}$, Sebastian Randel ${ }^{2}$, Wolfgang Freude ${ }^{2}$, Christian Koos ${ }^{1,2}$ \\ ${ }^{I}$ Institute of Microstructure Technology (IMT), Karlsruhe Institute of Technology (KIT), Germany \\ ${ }^{2}$ Institute of Photonics and Quantum Electronics (IPQ), Karlsruhe Institute of Technology (KIT), Germany \\ ${ }^{3}$ Institute for Applied Computer Science (IAI), Karlsruhe Institute of Technology (KIT), Germany \\ Authore-mail address: matthias.blaicher@kit.edu, christian.koos@kit.edu
}

\begin{abstract}
We demonstrate a 3D printed ultra-broadband and highly efficient out-of-plane coupler for photonic integrated circuits. The coupling efficiency at $\lambda=1550 \mathrm{~nm}$ is $-0.8 \mathrm{~dB}$ with a $1 \mathrm{~dB}$ bandwidth exceeding $100 \mathrm{~nm}$.
\end{abstract}

OCIS codes: (130.3120) Integrated optics devices; (130.5460) Polymer waveguides; (220.4000)

Microstructure fabrication

\section{Introduction}

Coupling fiber-guided light from or to on-chip waveguides is a key challenge of photonic integration. Out-of-plane coupling via the chip surface represents a particularly attractive approach, offering relaxed alignment tolerances along with scalability to large port counts while greatly simplifying routing of on-chip waveguides. For large-scale silicon-based photonic integrated circuits (PIC), out-of-plane coupling largely relies on grating couplers (GC) as the prevailing technical implementation [1,2]. However, despite tremendous progress in optimizing GC efficiency [3], the optical bandwidth is inherently limited to typical values between $40 \mathrm{~nm}$ and $50 \mathrm{~nm}$ due to the diffractive nature of the structures [4].

In this paper, we introduce a novel approach to highly efficient ultra-broadband out-of-plane coupling. The concept relies on 3D-printed freeform micro-lenses that are connected to on-chip waveguides by photonic wire bonds (PWB) [5]. These lensed PWB (LPWB) exploit the full flexibility of 3D printing and allow for efficient coupling of on-chip waveguides to a wide range of field profiles, thereby offering the possibility to significantly relax alignment tolerances. LPWB can be fabricated in fully automated processes at any position on the chip surface, and the propagation of the received or emitted light can have any orientation with respect to the surface normal. We prove the viability of the concept by coupling silicon strip waveguides to standard single-mode fibers (SSMF). At a center wavelength of $1550 \mathrm{~nm}$, we achieve a coupling efficiency of $-0.8 \mathrm{~dB}$ along with a $1 \mathrm{~dB}$ bandwidth well above $100 \mathrm{~nm}$.
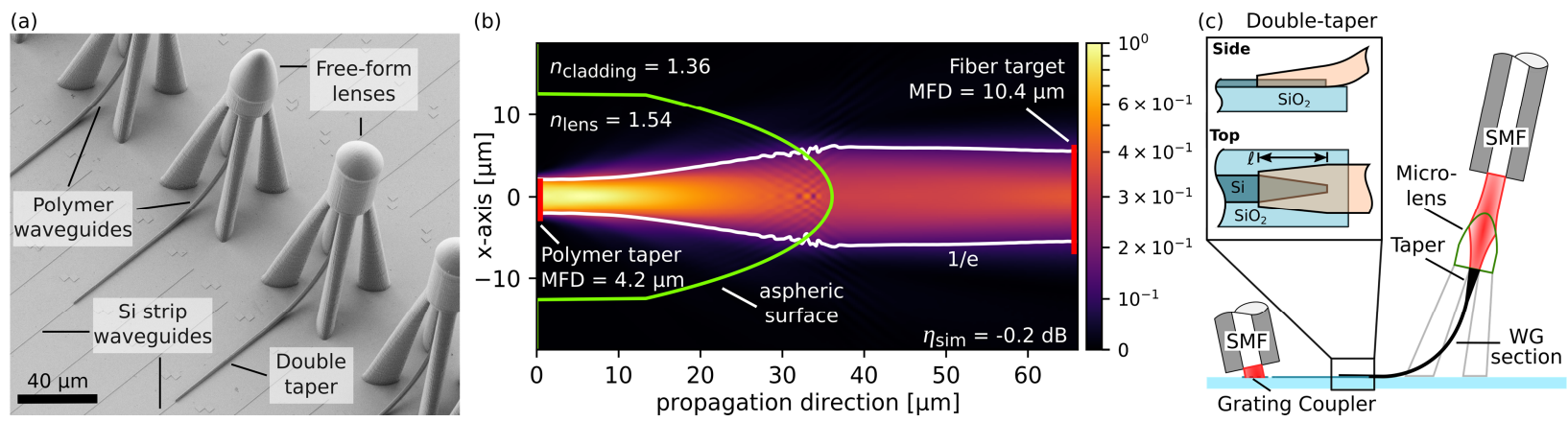

Fig. 1. Lensed photonic wire bonds (LPWB) as out-of-plane couplers. (a) Scanning electron micrograph of 3D microprinted out-of-plane couplers, comprising a dielectric polymer waveguide connected to a silicon nanowire on one side, and a microlens on the other side. The full structure is fabricated in a single lithographic step. The coupler axis is tilted by $12^{\circ}$ with respect to the chip normal in order to facilitate an easy comparison with GC which are also available on the chip. The concept can be readily adapted to vertically oriented coupler axes, too [6]. The micro-lens surface can be optimized for coupling to a large range of target mode fields. The printed structure is embedded in a low-index cladding (not shown). This helps in achieving single-mode operation of the PWB and low reflection at any optically relevant surfaces. (b) Simulated field [7] between polymer taper (see also subfigure (c)) and target SSMF, placed at a distance of $30 \mu \mathrm{m}$ from the lens surface. Taper and lens are immersed in oil, which could be replaced by a low-index cladding polymer. The simulated coupling efficiency of the lens (excluding Fresnel reflections) amounts to $\eta_{\mathrm{sim}}=-0.2 \mathrm{~dB}$ at $1550 \mathrm{~nm}$. The white contour line marks the radial $1 / \mathrm{e}^{2}$ intensity drop. (c) Schematic drawing of the coupling setup for characterizing lensed photonic wire bonds. Light is coupled into the silicon nanowire by a grating coupler. The waveguide (WG) section of the photonic wire bond is attached to the Si waveguide by a double-taper structure with a length of $l=60 \mu \mathrm{m}$. 


\section{Design and fabrication}

The LPWB comprises a typical silicon-to-polymer waveguide transition in form of a double-taper [5], the photonic wire bond, another taper attached to the lens along with the support structure, Fig. 1(a) and (c). The polymer WG features a cross section of $2 \mu \mathrm{m} \times 1.8 \mu \mathrm{m}$, combining mechanical stability with single-mode operation, and guides light from the on-chip strip WG close to the rear focal plane of the lens. The WG is tapered to a square cross-section of $5 \mu \mathrm{m} \times 5 \mu \mathrm{m}$ before entering the free-form lens, leading to a moderate beam divergence inside the lens. To achieve single-mode operation for the polymer waveguide and reduced Fresnel reflection at the lens and fiber surfaces, the whole setup is immersed in a low-refractive index oil with refractive index $n_{\text {cladding }}=1.36$. In a technical application, the oil would be replaced by a low-index cladding material that protects the structure.

The axis orientation, working distance and target mode field size of the micro-lens can be freely chosen. We designed our lens for focusing into a SSMF placed at a distance of $30 \mu \mathrm{m}$ from the lens apex. The rotationally symmetric lens surface is described by a fourth-order polynomial, the coefficients of which were numerically optimized with the help of a wide-angle, unidirectional wave propagation method [7]. A simulation of the electric field magnitude inside the lens section of the LPWB is depicted in Fig. 1(b).

The polymer structures were fabricated in situ by a single two-photon lithography step in a negative-tone photoresist (IP-Dip, Nanoscribe GmbH). Fully automated fabrication is enabled by detecting position and orientation of the silicon taper. To this end, we combine the data output from an observation camera as well as the data of a confocal detection unit of the 3D lithography system. The fabrication time of a single structure currently amounts to $7 \mathrm{~min}$, with vast potential for speed improvement by more than an order of magnitude. The fabricated structure is developed in propylene-glycol-methyl-ether-acetate (PGMEA), flushed with isopropanol, and subsequently blow dried. The LPWB were fabricated on a standard silicon chip processed in a CMOS line using $193 \mathrm{~nm}$ deep-ultraviolet lithography and a silicon device layer thickness $220 \mathrm{~nm}$. The tip widths of the inverse tapers amount to typically $120 \mathrm{~nm}$.

\section{Device characterization}

The LPWB couplers were attached to Si strip waveguides, which were connected to standard GC at the other end, Fig. 1(c), and the transmission is measured using a tunable laser source (TLS). The coupling efficiency of the LPWB coupler is then determined by using multiple on-chip GC-GC test structures as a reference. Figure 2 depicts the total measured Si-to-fiber coupling efficiency for 4 nominally identical LPWBs, including both the lens-to-SMF interface and the transition of the PWB to the on-chip waveguide. Three of the four structures exhibit a coupling efficiency better than $-1 \mathrm{~dB}$. Note that the optical bandwidth of our measurements is limited by the GC as well as by the tuning range of the TLS. Extrapolating the measurement results, we expect that the $1 \mathrm{~dB}$ bandwidth is well above $100 \mathrm{~nm}$, thereby outperforming even advanced GC designs. The optimum working distance between the LPWB lens apex and the SSMF agreed with simulations within $\pm 5 \mu \mathrm{m}$.

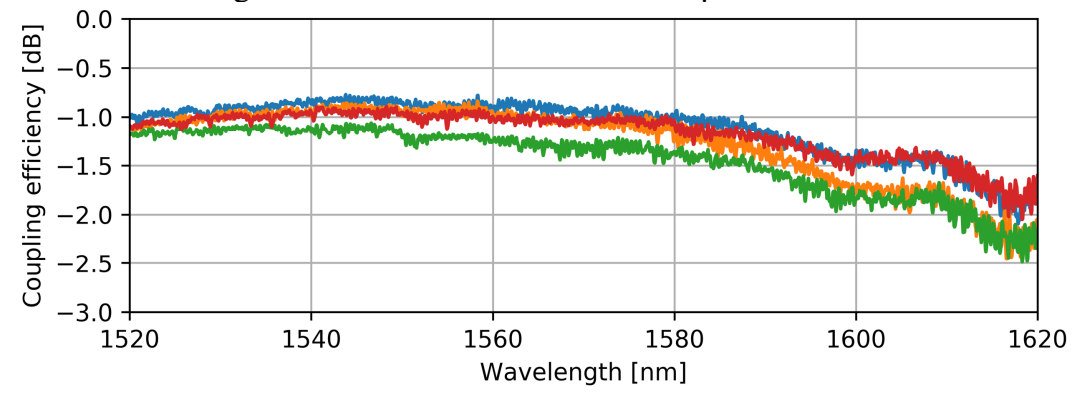

Fig. 2 Measured coupling efficiency of a coupling structure between a Si WG and a SMF fiber. Four nominally identical LPWB were investigated. The wavelength range is limited by the bandwidth of the silicon grating coupler, as well as by the tuning range of the laser source. Within these limits, the $1 \mathrm{~dB}$ bandwidth was found to be in excess of $100 \mathrm{~nm}$.

\section{Summary}

We demonstrate a 3D printed ultra-broadband and highly efficient out-of-plane coupler for photonic integrated circuits. The coupling efficiency at $\lambda=1550 \mathrm{~nm}$ is $-0.8 \mathrm{~dB}$ with a $1 \mathrm{~dB}$ bandwidth exceeding $100 \mathrm{~nm}$.

This work was supported by the EU-FP7 project BigPIPES, European Research Council (ERC Starting Grant 'EnTeraPIC' (\#280145), BMBF joint project PHOIBOS (Grant 13N12574) and PRIMA (13N14630), by the H2020 Photonic Packaging Pilot Line PIXAPP (\#731954), by the Alfried Krupp von Bohlen und Halbach Foundation, the Helmholtz International Research School for Teratronics (HIRST), the Karlsruhe School of Optics \& Photonics (KSOP), and the Karlsruhe Nano-Micro Facility (KNMF).

\section{References}

[1] D. Taillaert et al., Jpn. J. Appl. Phys. 45, no.8A, (2006): 6071-6077

[2] Yunhong Ding et al., 2013 Conf. Lasers Electro-Optics Pacific Rim 38, no.15, (2013): 1-2

[3] S. Scheerlinck et al., 2007 Conf. Lasers Electro-Optics 15, no.15, (2007): 1-2
Z. Xiao et al., Opt. Express 21, no.5, (2013): 5688

N. Lindenmann et al., J. Lightw. Technol. 33, no.4, (2015): 755-760

L. Zhu et al., Opt. Express 25, no.15, (2017): 18462

S. Schmidt et al., Opt. Express 24, no.26, (2016): 30188 